\title{
ON AN INEQUALITY BETWEEN DIRICHLET AND NEUMANN EIGENVALUES FOR THE LAPLACE OPERATOR
}

\author{
N. FILONOV
}

Dedicated to my teacher Mikhail Shlemovich Birman

\begin{abstract}
A simple proof of the inequality $\mu_{k+1}<\lambda_{k}$ is given. Here the $\lambda_{k}$ (respectively, $\mu_{k}$ ) are the eigenvalues of the Dirichlet (respectively, Neumann) problem for the Laplace operator in an arbitrary domain of finite measure in $\mathbb{R}^{d}, d>1$.
\end{abstract}

Let $\Omega$ be a domain in $\mathbb{R}^{d}$ such that the Sobolev space $W_{2}^{1}(\Omega)$ is compactly embedded in $L_{2}(\Omega)$. Then the spectra of the Dirichlet problem and the Neumann problem for the Laplace operator are both discrete. We denote the respective operators by $-\Delta_{\mathfrak{D}}$ and $-\Delta_{\mathfrak{N}}$, and enumerate their eigenvalues in increasing order (with multiplicity taken into account):

Note that

$$
\sigma\left(-\Delta_{\mathfrak{D}}\right)=\left\{\lambda_{k}\right\}_{k=1}^{\infty}, \quad \sigma\left(-\Delta_{\mathfrak{N}}\right)=\left\{\mu_{k}\right\}_{k=1}^{\infty}
$$

1) $\mu_{1}=0$ if the measure of the domain $\Omega$ is finite;

2) $\mu_{k+1}=\lambda_{k}$ if $d=1$

3) the inequality $\mu_{k} \leq \lambda_{k}$ is deduced immediately by variational arguments.

For $d=2$ and for a domain bounded by an analytic curve, Pólya and Szegő proved (see $\left[\mathrm{P},[\mathrm{S}]\right.$ ) that $\mu_{2} \leq \gamma \lambda_{1}$, where $\gamma$ is an absolute constant less than one (expressed in terms of zeros of Bessel functions). Since their proofs involve conformal mappings, they do not work in higher dimensions. Developing an idea used in [Pa], Levine and Weinberger established (see [LW]), for an arbitrary dimension, a series of inequalities of the form $\mu_{k+r}<\lambda_{k}, r=1, \ldots, d$, under some conditions on the principal curvatures of the $C^{2+\alpha}$-smooth boundary $\partial \Omega$ of a bounded domain $\Omega$. In particular, $\mu_{k+1}<\lambda_{k}$ if the mean curvature is nonnegative, and $\mu_{k+d} \leq \lambda_{k}$ for all convex domains.

Friedlander [F] proved the inequality $\mu_{k+1} \leq \lambda_{k}$ for the bounded domains $\Omega$ with $\partial \Omega \in$ $C^{1}$. He used the "Dirichlet-to-Neumann" operator $R(\lambda)$ that maps a function $\varphi$ defined on $\partial \Omega$ to the normal derivative on $\partial \Omega$ of the solution $u$ of the problem $(-\Delta-\lambda) u=0$ in $\Omega, u=\varphi$ on $\partial \Omega$. Friedlander obtained the following formula:

$$
n(\lambda)=N_{\mathfrak{N}}(\lambda)-N_{\mathfrak{D}}(\lambda) \quad \text { for } \lambda \notin \sigma\left(-\Delta_{\mathfrak{D}}\right) \cup \sigma\left(-\Delta_{\mathfrak{N}}\right),
$$

where $n(\lambda)$ is the number of negative eigenvalues of the operator $R(\lambda)$, and $N_{\mathfrak{N}}, N_{\mathfrak{D}}$ are the counting functions of the Laplace operator (see (1)), and he showed that $n(\lambda) \geq 1$, which implies the desired inequality.

We have succeeded in finding a simple proof of the inequality $\mu_{k+1}<\lambda_{k}$ in a more general situation.

2000 Mathematics Subject Classification. Primary 35J05, 35P15.

Key words and phrases. Dirichlet problem, Neumann problem, spectrum.

Supported by RFBR (grant no. 02-01-00798). 
Theorem. Suppose $d \geq 2$, a domain $\Omega \subset \mathbb{R}^{d}$ is such that the embedding $W_{2}^{1}(\Omega) \subset L_{2}(\Omega)$ is compact, and the measure of $\Omega$ is finite, $|\Omega|<\infty$. Then $\mu_{k+1}<\lambda_{k}$ for all $k$, where $\lambda_{k}$, $\mu_{k}$ are the eigenvalues of the Dirichlet problem and the Neumann problem, respectively.

The following simple fact will be used.

Lemma. For all $\mu$ we have

$$
\stackrel{\circ}{W}_{2}^{1}(\Omega) \cap \operatorname{ker}\left(-\Delta_{\mathfrak{N}}-\mu\right)=\{0\} .
$$

Proof. Let $v \in \stackrel{\circ}{W}_{2}^{1}(\Omega) \cap \operatorname{ker}\left(-\Delta_{\mathfrak{N}}-\mu\right)$. Since the function

$$
w(x)= \begin{cases}v(x) & \text { if } x \in \Omega, \\ 0 & \text { if } x \notin \Omega,\end{cases}
$$

belongs to $W_{2}^{1}\left(\mathbb{R}^{d}\right)$, we have

$$
\int_{\mathbb{R}^{d}}\langle\nabla w, \nabla \psi\rangle d x=\int_{\Omega}\langle\nabla v, \nabla \psi\rangle d x=-\mu \int_{\Omega} v \bar{\psi} d x=-\mu \int_{\mathbb{R}^{d}} w \bar{\psi} d x, \quad \psi \in C_{0}^{\infty}\left(\mathbb{R}^{d}\right),
$$

where the angle brackets denote the scalar product in $\mathbb{C}^{d}$. This implies that $-\Delta w=\mu w$, whence $w=0$.

Proof of the theorem. Let $N_{\mathfrak{D}}$ and $N_{\mathfrak{N}}$ denote the counting functions of the operators under consideration:

$$
N_{\mathfrak{D}}(\mu)=\operatorname{card}\left(\sigma\left(-\Delta_{\mathfrak{D}}\right) \cap[0, \mu]\right), \quad N_{\mathfrak{N}}(\mu)=\operatorname{card}\left(\sigma\left(-\Delta_{\mathfrak{N}}\right) \cap[0, \mu]\right) .
$$

It is known that

$$
\begin{aligned}
& N_{\mathfrak{D}}(\mu)=\max \left\{\operatorname{dim} L: L \subset \stackrel{\circ}{W_{2}^{1}}(\Omega), \int_{\Omega}|\nabla u|^{2} d x \leq \mu \int_{\Omega}|u|^{2} d x, u \in L\right\}, \\
& N_{\mathfrak{N}}(\mu)=\max \left\{\operatorname{dim} L: L \subset W_{2}^{1}(\Omega), \int_{\Omega}|\nabla u|^{2} d x \leq \mu \int_{\Omega}|u|^{2} d x, u \in L\right\} .
\end{aligned}
$$

We fix a positive number $\mu$ and take a subspace $F$ of $\stackrel{\circ}{W}_{2}^{1}(\Omega)$ such that $\operatorname{dim} F=N_{\mathfrak{D}}(\mu)$ and

$$
\int_{\Omega}|\nabla u|^{2} d x \leq \mu \int_{\Omega}|u|^{2} d x, \quad u \in F .
$$

The lemma implies that the sum $F \dot{+} \operatorname{ker}\left(-\Delta_{\mathfrak{N}}-\mu\right)$ is direct. Consider the set of functions $\left\{e^{i \omega x}\right\}_{|\omega|^{2}=\mu}$. Since all these functions are linearly independent, there exists a vector $\omega \in \mathbb{R}^{d},|\omega|=\sqrt{\mu}$, such that the function $e^{i \omega x}$ does not belong to $F \dot{+} \operatorname{ker}\left(-\Delta_{\mathfrak{N}}-\mu\right)$. Put

$$
G=F \dot{+} \operatorname{ker}\left(-\Delta_{\mathfrak{N}}-\mu\right) \dot{+}\left\{c e^{i \omega x}\right\}_{c \in \mathbb{C}}
$$

(if $\mu \notin \sigma\left(-\Delta_{\mathfrak{N}}\right)$, then $G$ is the sum of two terms). Clearly, $G \subset W_{2}^{1}(\Omega)$.

Let $\left(u+v+c e^{i \omega x}\right)$ be an element of $G, u \in F, v \in \operatorname{ker}\left(-\Delta_{\mathfrak{N}}-\mu\right)$. We have

$$
\begin{aligned}
\int_{\Omega}\left|\nabla\left(u+v+c e^{i \omega x}\right)\right|^{2} d x= & \int_{\Omega}\left(|\nabla u|^{2}+|\nabla v|^{2}+|c \omega|^{2}\right) d x \\
& +2 \operatorname{Re} \int_{\Omega}\left(\left\langle\nabla v, \nabla\left(u+c e^{i \omega x}\right)\right\rangle+\left\langle\nabla\left(c e^{i \omega x}\right), \nabla u\right\rangle\right) d x \\
= & : I_{1}+I_{2},
\end{aligned}
$$

and, by the definition of $F$,

$$
I_{1} \leq \mu \int_{\Omega}\left(|u|^{2}+|v|^{2}+|c|^{2}\right) d x .
$$


Furthermore, we have

$$
\begin{aligned}
I_{2} & =-2 \operatorname{Re} \int_{\Omega}\left(\Delta v \overline{\left(u+c e^{i \omega x}\right)}+\Delta\left(c e^{i \omega x}\right) \bar{u}\right) d x \\
& =2 \mu \operatorname{Re} \int_{\Omega}\left(v \overline{\left(u+c e^{i \omega x}\right)}+c e^{i \omega x} \bar{u}\right) d x,
\end{aligned}
$$

whence

$$
\int_{\Omega}\left|\nabla\left(u+v+c e^{i \omega x}\right)\right|^{2} d x \leq \mu \int_{\Omega}\left|u+v+c e^{i \omega x}\right|^{2} d x .
$$

Therefore,

$$
N_{\mathfrak{N}}(\mu) \geq \operatorname{dim} G=N_{\mathfrak{D}}(\mu)+\operatorname{dim} \operatorname{ker}\left(-\Delta_{\mathfrak{N}}-\mu\right)+1,
$$

by (2) and (3).

Now we take $\mu=\lambda_{k}$. Then

$$
\operatorname{card}\left(\sigma\left(-\Delta_{\mathfrak{N}}\right) \cap[0, \mu)\right)=N_{\mathfrak{N}}(\mu)-\operatorname{dim} \operatorname{ker}\left(-\Delta_{\mathfrak{N}}-\mu\right) \geq N_{\mathfrak{D}}(\mu)+1 \geq k+1,
$$

which shows that $\mu_{k+1}<\lambda_{k}$.

Remark. Our construction is based on a function $f$ such that $-\Delta f=\mu f$ and $|\nabla f| \leq$ $\sqrt{\mu}|f|$. In the Euclidean space this is an exponential: $f(x)=e^{i \omega x}$. The question as to whether such a function exists on a manifold is not trivial. For example, if we cut a sphere into two parts by a plane that does not contain the center of the sphere, then the desired function fails to exist in the part containing a hemisphere. For some classes of manifolds such a function was constructed in $[\mathrm{M}]$.

\section{ADDED IN PROOF}

In the same way we can treat a more general case of a mixed boundary condition instead of the Neumann problem.

Theorem. Let $d \geq 2$, and let $\Omega$ be a bounded domain in $\mathbb{R}^{d}$ with Lipschitz boundary $\partial \Omega$. Suppose $\sigma$ is a real function on $\partial \Omega, \sigma \in L_{q}(\partial \Omega)$, where $q>1$ for $d=2$ and $q=d-1$ for $d>2$. Let $\mu_{k}^{\sigma}$ denote the eigenvalues of the problem

$$
-\Delta u=\mu_{k}^{\sigma} u \text { in } \Omega, \quad \frac{\partial u}{\partial n}+\sigma u=0 \text { on } \partial \Omega,
$$

where $n$ is the outward normal to the boundary. If $\int_{\partial \Omega} \sigma(x) d x \leq 0$, then $\mu_{k+1}^{\sigma}<\lambda_{k}$ for all $k$.

Remark. The problem $(*)$ corresponds to the quadratic form

$$
\int_{\Omega}|\nabla u|^{2} d x+\int_{\partial \Omega} \sigma|u|^{2} d x
$$

with domain $W_{2}^{1}(\Omega)$. For $\sigma=0$ this is a Neumann problem, $\mu_{k}^{0}=\mu_{k}$.

\section{REFERENCES}

[F] L. Friedlander, Some inequalities between Dirichlet and Neumann eigenvalues, Arch. Rational Mech. Anal. 116 (1991), 153-160. MF1143438 (93h:35146)

[LW] H. Levine and H. Weinberger, Inequalities between Dirichlet and Neumann eigenvalues, Arch. Rational Mech. Anal. 94 (1986), 193-208. MR0846060|(87k:35186)

[M] R. Mazzeo, Remarks on a paper of L. Friedlander concerning inequalities between Dirichlet and Neumann eigenvalues, Internat. Math. Res. Notices 1991, no. 4, 41-48. MR.1121164 (93h:35147)

[Pa] L. Payne, Inequalities for eigenvalues of membranes and plates, J. Rational Mech. Anal. 4 (1955), 517-529. MR0070834(17:42a) 
[P] G. Pólya, Remarks on the foregoing paper, J. Math. Physics 31 (1952), 55-57. MR0047237 $(13: 846 \mathrm{f})$

[S] G. Szegö, Inequalities for certain eigenvalues of a membrane of given area, J. Rational Mech. Anal. 3 (1954), 343-356. MR0061749 (15:877c)

St. Petersburg State University, Physics Department, Ulyanovskaya Ul. 1, Petrodvorets, St. Petersburg 198504, Russia

E-mail address: filonov@mph.phys.spbu.ru

Received 1/SEP/2003

Translated by THE AUTHOR 